\title{
Vacuum Quantum Effects for Parallel Plates Moving by Uniform Acceleration in Static de Sitter Space
}

\author{
M.R. Setare * \\ Physics Dept. Inst. for Studies in Theo. Physics and Mathematics(IPM) \\ P. O. Box 19395-5531, Tehran, IRAN
}

\begin{abstract}
The Casimir forces on two parallel plates moving by uniform proper acceleration in static de Sitter background due to conformally coupled massless scalar field satisfying Dirichlet boundary conditions on the plates is investigated. Static de Sitter space is conformally related to the Rindler space, as a result we can obtain vacuum expectation values of energy-momentum tensor for conformally invariant field in static de Sitter space from the corresponding Rindler counterpart by the conformal transformation.
\end{abstract}

\footnotetext{
*E-mail: rezakord@ipm.ir
} 


\section{Introduction}

The Casimir effect is one of the most interesting manifestations of nontrivial properties of the vacuum state in quantum field theory $[1,2]$. Since its first prediction by Casimir in 1948[2] this effect has been investigated for different fields having different boundary geometries[3-9]. The Casimir effect can be viewed as the polarization of vacuum by boundary conditions or geometry. Therefore, vacuum polarization induced by a gravitational field is also considered as Casimir effect.

Casimir stress for parallel plates in the background of static domain wall in four and two dimensions is calculated in $[10,11]$. Spherical bubbles immersed in different de Sitter spaces in- and out-side is calculated in $[12,13]$.

It is well known that the vacuum state for an uniformly accelerated observer, the FullingRindler vacuum $[14,15,16,17,18]$, turns out to be inequivalent to that for an inertial observer, the familiar Minkowski vacuum. Quantum field theory in accelerated systems contains many of special features produced by a gravitational field avoiding some of the difficulties entailed by renormalization in a curved spacetime. In particular, near the canonical horizon in the gravitational field, a static spacetime may be regarded as a Rindler-like spacetime. Rindler space is conformally related to the static de Sitter space and to the Robertson-Walker space with negative spatial curvature. As a result the expectation values of the energy-momentum tensor for a conformally invariant field and for corresponding conformally transformed boundaries on the de Sitter and RobertsonWalker backgrounds can be derived from the corresponding Rindler counterpart by the standard transformation [19]. The authors in [19] have shown that the Minkowski vacuum contains a thermal spectrum of Rindler particles. One can also demonstrate this by showing that the Green functions in Minkowski vacuum are Rindler thermal Green functions. In a similar way one can relate the vacua of static de Sitter space and de Sitter space have the same curvature, but static de Sitter space is a member of Rindler class, while de Sitter space is a member of Minkowski space.

In this paper we will study the scalar vacuum polarization brought about by the presence of infinite plane boundary moving by uniform acceleration through the static de Sitter vacuum. This problem for the conformally coupled Dirichlet and Neumann massless scalar and electromagnetic fields in four dimensional Rindler spacetime was considered by Candelas and Deutsch [20]. Here we will investigate the vacuum expectation values of the energy-momentum tensor for the massless scalar field with conformal curvature coupling and satisfying Dirichlet boundary condition on the infinite plane in four spacetime dimension. Here we use the results of Ref. [18] to generate vacuum energy-momentum tensor for the static de Sitter background which is conformally related to the Rindler spacetime. Previously this method has been used in [21] to drive the vacuum stress on parallel plates for scalar field with Dirichlet boundary condition in de Sitter space. Also this method has been used in [22] to derive the vacuum characteristics of the Casimir configuration on background of conformally flat brane-world geometries for massless scalar field with Robin boundary condition on the plates.

In section two we calculate the stress on two parallel plates with Dirichlet boundary conditions. The last section conclude and summarize the results. 


\section{Vacuum expectation values for the energy-momentum tensor}

We will consider a conformally coupled massless scalar field $\varphi(x)$ satisfying the following equation

$$
\left(\nabla_{\mu} \nabla^{\mu}+\frac{1}{6} R\right) \varphi(x)=0
$$

on the background of a static form of de Sitter space-time. In Eq. (1) $\nabla_{\mu}$ is the operator of the covariant derivative, and $R$ is the Ricci scalar for the de Sitter space.

$$
R=\frac{12}{\alpha^{2}}
$$

The static form of de Sitter space time which is conformally related to the Rindler space time is given by [19]

$$
d s^{2}=\left[1-\left(\frac{r^{2}}{\alpha^{2}}\right)\right] d t^{2}-\left[1-\left(\frac{r^{2}}{\alpha^{2}}\right)\right]^{-1} d r^{2}-r^{2}\left(d \theta^{2}+\sin ^{2} \theta d \phi^{2}\right),
$$

under the change of variable $\frac{r}{\alpha}=r^{\prime}\left(1+r^{2}\right)^{-1 / 2}$ we obtain

$$
d s^{2}=\left(\alpha^{2}-r^{2}\right)\left[\frac{d t^{2}}{\alpha^{2}}-\gamma d r^{\prime 2}-r^{\prime 2}\left(d \theta^{2}+\sin ^{2} \theta d \phi^{2}\right)\right]
$$

with $\gamma=\left(1+r^{\prime 2}\right)^{-1}$, which is manifestly conformal to following Rindler space time if $\eta=\frac{t}{\alpha}$

$$
d s^{2}=\zeta^{2}\left[d \eta^{2}-\gamma d r^{2}-r^{2}\left(d \theta^{2}+\sin ^{2} \theta d \phi^{2}\right)\right]=\xi^{2} d \tau^{2}-d \xi^{2}-d y^{2}-d z^{2},
$$

This metric can be written in coordinates $(\tau, r, \theta, \phi)$ related to the previous ones by transformation

$$
\xi=\frac{R}{1-R \cos \theta}, \quad y=\frac{R \sin \theta \cos \phi}{1-R \cos \theta}, \quad z=\frac{R \sin \theta \sin \phi}{1-R \cos \theta}
$$

where

$$
R=R(r)=\frac{r}{\sqrt{1+r^{2}}}
$$

In these coordinates the metric takes the form

$$
d s_{\text {Rind }}^{2}=\xi^{2}\left[d \tau^{2}-\frac{d r^{2}}{1+r^{2}}-r^{2}\left(d \theta^{2}+\sin ^{2} \theta d \phi^{2}\right)\right], \quad \xi=\xi(r, \theta) .
$$

Let us denote the energy-momentum tensor in coordinates $(\tau, \xi, y, z)$ as $T_{\mu \nu}$, and the same tensor in coordinates $(\tau, r, \theta, \phi)$ as $T_{\mu \nu}^{\prime}$. The tensor $T_{\mu \nu}$ has the structure

$$
T_{\mu}^{\nu}=\operatorname{diag}\left(\varepsilon,-p,-p_{\perp}, p_{\perp}\right) .
$$

By making use the standard transformation law for the second rank tensors, for the nonzero components in the new coordinate system one finds

$$
T_{r r}^{\prime}=R^{\prime 2}(r)\left(\frac{\partial \xi}{\partial R}\right)^{2}\left(T_{\xi \xi}+\sin ^{2} \theta T_{y y}\right)
$$




$$
\begin{aligned}
T_{r \theta}^{\prime} & =\frac{\partial \xi}{\partial R} R^{\prime}(r)\left[\frac{\partial \xi}{\partial \theta}\left(T_{\xi \xi}+\sin ^{2} \theta T_{y y}\right)+\xi \sin \theta \cos \theta T_{y y}\right] \\
T_{\theta \theta}^{\prime} & =\left(\frac{\partial \xi}{\partial \theta}\right)^{2} T_{\xi \xi}^{2}+\left[\frac{\partial}{\partial \theta}(\xi \sin \theta)\right]^{2} T_{y y} \\
T_{\phi \phi}^{\prime} & =\xi^{2} \sin ^{2} \theta T_{y y} .
\end{aligned}
$$

Our main interest in the present paper is to investigate the vacuum expectation values (VEV's) of the energy-momentum tensor for the field $\varphi(x)$ in the background of the above de Sitter space time induced by two parallel plates moving with uniform proper acceleration.we will consider the case of a scalar field satisfying Dirichlet boundary condition on the surface of the plates:

$$
\left.\varphi\right|_{\xi=\xi_{1}}=\left.\varphi\right|_{\xi=\xi_{2}}=0
$$

The presence of boundaries modifies the spectrum of the zero-point fluctuations compared to the case without boundaries. This results in the shift in the VEV's of the physical quantities, such as vacuum energy density and stresses. This is the well known Casimir effect. It can be shown that for a conformally coupled scalar by using field equation (1) the expression for the energy-momentum tensor can be presented in the form

$$
T_{\mu \nu}=\nabla_{\mu} \varphi \nabla_{\nu} \varphi-\frac{1}{6}\left[\frac{g_{\mu \nu}}{2} \nabla_{\rho} \nabla^{\rho}+\nabla_{\mu} \nabla_{\nu}+R_{\mu \nu}\right] \varphi^{2}
$$

where $R_{\mu \nu}$ is the Ricci tensor. The quantization of a scalar filed on background of metric Eq.(3) is standard. Let $\left\{\varphi_{\alpha}(x), \varphi_{\alpha}^{*}(x)\right\}$ be a complete set of orthonormalized positive and negative frequency solutions to the field equation (1), obying boundary condition (14). By expanding the field operator over these eigenfunctions, using the standard commutation rules and the definition of the vacuum state for the vacuum expectation values of the energy-momentum tensor one obtains

$$
\left\langle 0\left|T_{\mu \nu}(x)\right| 0\right\rangle=\sum_{\alpha} T_{\mu \nu}\left\{\varphi_{\alpha}, \varphi_{\alpha}^{*}\right\}
$$

where $|0\rangle$ is the amplitude for the corresponding vacuum state, and the bilinear form $T_{\mu \nu}\{\varphi, \psi\}$ on the right is determined by the classical energy-momentum tensor (15). Instead of evaluating Eq. (16) directly on background of the curved metric, the vacuum expectation values can be obtained from the corresponding Rindler space time results for a scalar field $\bar{\varphi}$ by using the conformal properties of the problem under consideration. Under the conformal transformation $g_{\mu \nu}=\Omega^{2} \bar{g}_{\mu \nu}$ the $\bar{\varphi}$ field will change by the rule

$$
\varphi(x)=\Omega^{-1} \bar{\varphi}(x),
$$

where for metric Eq.(3) the conformal factor is given by $\Omega=\frac{\sqrt{\alpha^{2}-r^{2}}}{\xi}$. The Casimir effect with boundary conditions (14) on two parallel plates moving with uniform proper acceleration on background of the Rindler spacetime is investigated in Ref. [18] for a scalar field with a Dirichlet and Neumann boundary condition. In the case of a conformally coupled scalar the corresponding regularized VEV's for the energy-momentum tensor in the region between the plates have the form

$$
\left\langle 0_{D}\left|T_{i}^{k}\right| 0_{D}\right\rangle=A_{3} \delta_{i}^{k} \int_{0}^{\infty} d k k^{3} \int_{0}^{\infty} d \omega\left\{\frac{\sinh \pi \omega}{\pi} f^{(i)}\left[\tilde{D}_{i \omega}\left(k \xi, k \xi_{2}\right)\right]-\frac{I_{\omega}\left(k \xi_{1}\right)}{I_{\omega}\left(k \xi_{2}\right)} \frac{F^{(i)}\left[D_{\omega}\left(k \xi, k \xi_{2}\right)\right]}{D_{\omega}\left(k \xi_{1}, k \xi_{2}\right)}\right\},
$$


where $\left|0_{D}\right\rangle$ is the amplitude for the Dirichlet vacuum between the plates, and

$$
A_{3}=\frac{1}{2 \pi^{2}}
$$

also we have introduced the notation

$$
\tilde{D}_{i \omega}\left(k \xi, k \xi_{2}\right)=K_{i \omega}(k \xi)-\frac{K_{i \omega}\left(k \xi_{2}\right)}{I_{i \omega}\left(k \xi_{2}\right)} I_{i \omega}(k \xi),
$$

and the functions $F^{(i)}[G(z)], i=0,1,2,3$ are as following

$$
F^{(i)}[G(z)]=f^{(i)}[G(z), \omega \rightarrow i \omega] .
$$

here for a given function $G(z)$ we use the notations

$$
\begin{aligned}
f^{(0)}[G(z)] & =\frac{1}{6}\left|\frac{d G(z)}{d z}\right|^{2}+\frac{1}{6 z} \frac{d}{d z}|G(z)|^{2}+\frac{1}{6}\left[1+5 \frac{\omega^{2}}{z^{2}}\right]|G(z)|^{2}, \\
f^{(1)}[G(z)] & =-\frac{1}{2}\left|\frac{d G(z)}{d z}\right|^{2}-\frac{1}{6 z} \frac{d}{d z}|G(z)|^{2}+\frac{1}{2}\left(1-\frac{\omega^{2}}{z^{2}}\right)|G(z)|^{2} \\
f^{(i)}[G(z)] & =-\frac{|G(z)|^{2}}{2}+\frac{1}{6}\left[\left|\frac{d G(z)}{d z}\right|^{2}+\left(1-\frac{\omega^{2}}{z^{2}}\right)|G(z)|^{2}\right] ; \quad i=2,3
\end{aligned}
$$

where $G(z)=D_{i \omega}\left(z, k \xi_{2}\right)$, which given by following expression, and the indices 0,1 correspond to the coordinates $\tau, \xi$ respectively,

$$
D_{i \omega}\left(k \xi, k \xi_{2}\right)=I_{i \omega}\left(k \xi_{2}\right) K_{i \omega}(k \xi)-K_{i \omega}\left(k \xi_{2}\right) I_{i \omega}(k \xi) .
$$

Now let us present the VEV's (18) in the form

$$
\left\langle 0\left|T_{i}^{k}\right| 0\right\rangle_{D}=\left\langle 0_{R}\left|T_{i}^{k}\right| 0_{R}\right\rangle+\left\langle T_{i}^{k}\right\rangle_{D}^{(1 b)}\left(\xi_{1}, \xi\right)+\left\langle T_{i}^{k}\right\rangle_{D}^{(1 b)}\left(\xi_{2}, \xi\right)+\Delta\left\langle T_{i}^{k}\right\rangle_{D}\left(\xi_{1}, \xi_{2}, \xi\right), \quad \xi_{1}<\xi<\xi_{2},
$$

where

$$
\left\langle 0_{R}\left|T_{i}^{k}\right| 0_{R}\right\rangle=\frac{A_{3} \delta_{i}^{k}}{\pi} \int_{0}^{\infty} d k k^{3} \int_{0}^{\infty} d \omega \sinh \pi \omega f^{(i)}\left[K_{i \omega}(k \xi)\right]
$$

are the corresponding VEV's for the Fulling-Rindler vacuum without boundaries, all divergences are contained in this part. These divergences can be regularized subtracting the corresponding VEV's for the Minkowskian vacuum. The subtracted VEV's

$$
\left\langle T_{i}^{k}\right\rangle_{\mathrm{sub}}^{(R)}=\left\langle 0_{R}\left|T_{i}^{k}\right| 0_{R}\right\rangle-\left\langle 0_{M}\left|T_{i}^{k}\right| 0_{M}\right\rangle
$$

are investigated in a large number of papers, [18, 20, 23, 24, 25, 26, 27, 28, 29, 30, 31, 32, $33,34]$. For a massless scalar VEV's (28) can be presented in the form

$$
\left\langle T_{i}^{k}\right\rangle_{\mathrm{sub}}^{(R)}=-\frac{\delta_{i}^{k} \xi^{-d-1}}{2^{d-1} \pi^{d / 2} \Gamma(d / 2)} \int_{0}^{\infty} \frac{\omega^{d} g^{(i)}(\omega) d \omega}{e^{2 \pi \omega}+(-1)^{d}}
$$

(the expressions for the functions $g^{(i)}(\omega)$ are given in Ref. [23]) correspond to the absence from the vacuum of thermal distribution with standard temperature $T=(2 \pi \xi)^{-1}$. As we see from Eq. (29), the corresponding spectrum has non- Planckian form: the density of 
states factor is not proportional to $\omega^{d-1} d \omega$ where $d$ is dimension of space. The spectrum takes the Planckian form for conformally coupled scalars in $d=1,2,3$ with $g^{(0)}(\omega)=$ $-d g^{(i)}(\omega)=1, i=1,2, \ldots d$. In four dimensional space time $d=3$ we have

$$
\left\langle T_{i}^{k}\right\rangle_{\mathrm{sub}}^{(R)}=\frac{-1}{480 \pi^{2} \xi^{4}} \operatorname{diag}(1,-1 / 3,-1 / 3,-1 / 3)
$$

The boundary part term $\left\langle T_{i}^{k}\right\rangle_{D}^{(1 b)}\left(\xi_{1}, \xi\right)$ are give by

$$
\left\langle T_{i}^{k}\right\rangle_{D}^{(1 b)}\left(\xi_{1}, \xi\right)=-A_{3} \delta_{i}^{k} \int_{0}^{\infty} d k k^{3} \int_{0}^{\infty} d \omega \frac{I_{\omega}\left(k \xi_{1}\right)}{K_{\omega}\left(k \xi_{1}\right)} F^{(i)}\left[K_{\omega}(k \xi)\right]
$$

is induced in the region $\xi>\xi_{1}$ by the presence of a single plane boundary located at $\xi=\xi_{1}$. The expressions for the boundary part $\left\langle T_{i}^{k}\right\rangle_{D}^{(1 b)}\left(\xi_{2}, \xi\right)$ in the region $\xi<\xi_{2}$ are obtained from formulae (31) by replacing

$$
I_{\omega} \rightarrow K_{\omega}, \quad K_{\omega} \rightarrow I_{\omega}, \quad \xi_{1} \rightarrow \xi_{2}, \quad \xi_{2} \rightarrow \xi_{1}
$$

Also

$$
\Delta\left\langle T_{i}^{k}\right\rangle_{D}=-A_{3} \delta_{i}^{k} \int_{0}^{\infty} d k k^{3} \int_{0}^{\infty} d \omega I_{\omega}\left(k \xi_{1}\right)\left[\frac{F^{(i)}\left[D_{\omega}\left(k \xi, k \xi_{2}\right)\right]}{I_{\omega}\left(k \xi_{2}\right) D_{\omega}\left(k \xi_{1}, k \xi_{2}\right)}-\frac{F^{(i)}\left[K_{\omega}(k \xi)\right]}{K_{\omega}\left(k \xi_{1}\right)}\right]
$$

is the 'interference' term.

The vacuum energy-momentum tensor on static de Sitter space (sd) Eq.(3) is obtained by the standard transformation law between conformally related problems (see, for instance, [19]) and has the form

$$
\left\langle T_{\nu}^{\mu}\left[g_{\alpha \beta}\right]\right\rangle_{\mathrm{sd}}=\xi^{4}\left(\alpha^{2}-r^{2}\right)^{-2}\left\langle T_{\nu}^{\prime \mu}\left[g_{\alpha \beta}\right]\right\rangle_{\text {Rindler }}+\left\langle T_{\nu}^{\mu}\left[g_{\alpha \beta}\right]\right\rangle_{\mathrm{ss}}
$$

Here the first term on the right is the vacuum energy-momentum tensor for Rindler space in coordinates $(\tau, r, \theta, \phi)$, and the second one is the situation without boundaries (pure gravitational part). The contribution from pure gravitational part is independent of coordinates, and so will be the same as the stady-state case(ss) [19].

The second term in Eq.(34) can be rewritten as following

$$
\left\langle T_{\nu}^{\mu}\left[g_{\alpha \beta}\right]\right\rangle_{\mathrm{ss}}=-\frac{1}{2880}\left[\frac{1}{6} \tilde{H}_{\nu}^{(1) \mu}-\tilde{H}_{\nu}^{(3) \mu}\right]
$$

The functions $H_{\nu}^{(1,3) \mu}$ are some combinations of curvature tensor components (see [19]). For massless scalar field in de Sitter space (steady state), the term is given by [19, 35]

$$
-\frac{1}{2880}\left[\frac{1}{6} \tilde{H}_{\nu}^{(1) \mu}-\tilde{H}_{\nu}^{(3) \mu}\right]=\frac{1}{960 \pi^{2} \alpha^{4}} \delta_{\nu}^{\mu} .
$$

Therefore the vacuum energy-momentum tensor (34) has the form

$$
\left\langle T_{\nu}^{\mu}\left[g_{\alpha \beta}\right]\right\rangle_{\mathrm{ren}}=\left\langle T_{\nu}^{\mu}\left[g_{\alpha \beta}\right]\right\rangle_{\mathrm{ren}}^{(0)}+\left\langle T_{\nu}^{\mu}\left[g_{\alpha \beta}\right]\right\rangle_{\mathrm{ren}}^{(b)}
$$

Where the first term on the right is the vacuum energy-momentum tensor for the situation without boundaries (gravitational part), and the second one is due to the presence 
of boundaries. By taking into account Eqs.(30,34, 36) the first term in Eq.(37)can be rewritten as following

$$
\left\langle T_{\nu}^{\mu}\left[g_{\alpha \beta}\right]\right\rangle_{\mathrm{ren}}^{(0)}=\frac{-1}{480 \pi^{2}}\left(\alpha^{2}-r^{2}\right)^{-2} \operatorname{diag}(1,-1 / 3,-1 / 3,-1 / 3)+\frac{1}{960 \pi^{2} \alpha^{4}} \delta_{\nu}^{\mu} .
$$

The boundary part in Eq.(37) is related to the corresponding Rindler spacetime counterpart Eq.(26) by the relation

$$
\left\langle T_{\nu}^{\mu}\left[g_{\alpha \beta}\right]\right\rangle_{\text {ren }}^{(b)}=\xi^{4}\left(\alpha^{2}-r^{2}\right)^{-2}\left(\left\langle T_{i}^{k}\right\rangle_{D}^{(1 b)}\left(\xi_{1}, \xi\right)+\left\langle T_{i}^{k}\right\rangle_{D}^{(1 b)}\left(\xi_{2}, \xi\right)+\Delta\left\langle T_{i}^{k}\right\rangle_{D}\left(\xi_{1}, \xi_{2}, \xi\right)\right), \quad \xi_{1}<\xi<\xi_{2} .
$$

Now we turn to the interaction forces between the plates. The vacuum force acting per unit surface of the plate at $\xi=\xi_{i}$ is determined by the ${ }_{1}^{1}$-component of the vacuum EMT at this point. The gravitational part of the pressure according to Eq.(38) is equal to

$$
P_{g}=-<T_{1}^{1}>=\frac{-1}{960 \pi^{2} \alpha^{4}}-\frac{1}{1440 \pi^{2}}\left(\alpha^{2}-r^{2}\right)^{-2} .
$$

The first term is the same from both sides of the plates, and hence leads to zero effective force. The corresponding effective boundary part pressures can be presented as a sum of two terms (at first we consider boundary part pressures in Rindler spacetime)

$$
p_{b}^{(i)}=p_{b 1}^{(i)}+p_{b(\mathrm{int})}^{(i)}, \quad i=1,2 .
$$

The first term on the right is the pressure for a single plate at $\xi=\xi_{i}$ when the second plate is absent. This term is divergent due to the well known surface divergences in the subtracted VEV's. The second term on the right of Eq. (41),

$$
p_{b(\mathrm{int})}^{(i)}=-\left\langle T_{1}^{1}\right\rangle_{b}^{(1 b)}\left(\xi_{j}, \xi_{i}\right)-\Delta\left\langle T_{1}^{1}\right\rangle_{b}\left(\xi_{1}, \xi_{2}, \xi_{i}\right), \quad i, j=1,2, \quad j \neq i
$$

is the pressure induced by the presence of the second plate, and can be termed as an interaction force. For the plate at $\xi=\xi_{2}$ the interaction term is due to the second summand on the right of Eq. (18). Substituting into this term $\xi=\xi_{2}$ and using the Wronskian relation for the modified Bessel functions one has

$$
p_{b(\mathrm{int})}^{(2)}\left(\xi_{1}, \xi_{2}\right)=-\frac{A_{3}}{2 \xi_{2}^{2}} \int_{0}^{\infty} d k k \int_{0}^{\infty} d \omega \frac{I_{\omega}\left(k \xi_{1}\right)}{I_{\omega}\left(k \xi_{2}\right) D_{\omega}\left(k \xi_{1}, k \xi_{2}\right)} .
$$

By a similar way for the interaction term on the plate at $\xi=\xi_{1}$ we obtain

$$
p_{b(\mathrm{int})}^{(1)}\left(\xi_{1}, \xi_{2}\right)=-\frac{A_{3}}{2 \xi_{1}^{2}} \int_{0}^{\infty} d k k \int_{0}^{\infty} d \omega \frac{K_{\omega}\left(k \xi_{2}\right)}{K_{\omega}\left(k \xi_{1}\right) D_{\omega}\left(k \xi_{1}, k \xi_{2}\right)} .
$$

In the limit $\xi_{2} \gg \xi_{1}$, introducing in Eq. (43) a new integration variable $x=k \xi_{2}$, and making use the formula

$$
I_{\omega}(y)=\left(\frac{y}{2}\right)^{\omega} \frac{1}{\Gamma(\omega)}\left[1+\mathcal{O}\left(y^{2}\right)\right], \quad y=x \xi_{1} / \xi_{2},
$$

and the standard relation between the functions $K_{\omega}$ and $I_{ \pm \omega}$ one finds

$$
p_{b(\text { int })}^{(2)} \approx-\frac{\pi^{2} A_{3}}{48 \xi_{2}^{4} \ln ^{2}\left(2 \xi_{2} / \xi_{1}\right)} \int_{0}^{\infty} \frac{d x x}{I_{0}^{2}(x)}\left[1+\mathcal{O}\left(\frac{\ln x}{\ln \left(2 \xi_{2} / \xi_{1}\right)}\right)\right] .
$$


The similar calculation for Eq. (44) yields

$$
p_{b(\mathrm{int})}^{(1)} \approx-\frac{\pi^{2} A_{3}}{24 \xi_{2} \xi_{1}^{2} \ln ^{3}\left(2 \xi_{2} / \xi_{1}\right)} \int_{0}^{\infty} \frac{d x x K_{0}(x)}{I_{0}(x)}\left[1+\mathcal{O}\left(\frac{\ln x}{\ln \left(2 \xi_{2} / \xi_{1}\right)}\right)\right] .
$$

Therefore the effective boundary part pressures in static de Sitter space acting on the plates are given by, $p_{b(\text { int })}^{1,2}=-\left\langle T_{1}^{1}\left[g_{\alpha \beta}\right]\right\rangle_{\mathrm{ren}}^{(b)}$, for the plate at $\xi=\xi_{2}$ we have

$$
p_{b(\mathrm{int})}^{(2)}\left(\xi_{1}, \xi_{2}\right)=-\xi_{2}^{2}\left(\alpha^{2}-r^{2}\right)^{-2} \frac{A_{3}}{2} \int_{0}^{\infty} d k k \int_{0}^{\infty} d \omega \frac{I_{\omega}\left(k \xi_{1}\right)}{I_{\omega}\left(k \xi_{2}\right) D_{\omega}\left(k \xi_{1}, k \xi_{2}\right)} .
$$

For the interaction term on the plate at $\xi=\xi_{1}$ we obtain

$$
p_{b(\text { int })}^{(1)}\left(\xi_{1}, \xi_{2}\right)=-\xi_{1}^{2}\left(\alpha^{2}-r^{2}\right)^{-2} \frac{A_{3}}{2} \int_{0}^{\infty} d k k \int_{0}^{\infty} d \omega \frac{K_{\omega}\left(k \xi_{2}\right)}{K_{\omega}\left(k \xi_{1}\right) D_{\omega}\left(k \xi_{1}, k \xi_{2}\right)} .
$$

As the function $D_{\omega}\left(k \xi, k \xi_{2}\right)$ is positive for $\xi_{1}<\xi_{2}$, interaction forces per unit surface Eqs.(48) and (49) are always attractive.

\section{Conclusion}

Quantum field theory in accelerated systems contains many of special features produced by a gravitational field avoiding some of the difficulties entailed by renormalization in a curved spacetime. In particular, the near horizon geometry of most black holes is well approximated by Rindler and a better understanding of physical effects in this background could serve as a handle to deal with more complicated geometries like Schwarzschild. The Rindler geometry shares most of the qualitative features of black holes and is simple enough to allow detailed analysis. Another motivation for the investigation of quantum effects in the Rindler space is related to the fact that this space is conformally related to the de Sitter space and to the Robertson-Walker space with negative spatial curvature. As a result the expectation values of the energy-momentum tensor for a conformally invariant field and for corresponding conformally transformed boundaries on the de Sitter and Robertson-Walker backgrounds can be derived from the corresponding Rindler counterpart by the standard transformation .

In the present paper we have investigated the Casimir effect for a conformally coupled massless scalar field between two parallel plates moving by uniform acceleration, on background of the static de Sitter spacetimes which is conformally related to the Rindler spacetime. We have assumed that the scalar field satisfies Dirichlet boundary condition on the plates. The vacuum expectation values of the energy-momentum tensor are derived from the corresponding Rindler spacetime results by using the conformal properties of the problem. As the boundaries are static in the Rindler coordinates no Rindler quanta are created. In the region between the plates the boundary induced part for the vacuum energy-momentum tensor is given by Eq.(39), and the corresponding vacuum forces acting per unit surface of the plates have the form Eqs. (48),(49). The vacuum polarization due to the gravitational field, without any boundary conditions is given by Eq.(38), the corresponding gravitational pressure part has the form Eq.(40), the first term in this equation is the same from both sides of the plates, and hence leads to zero effective force. Therefore the effective force acting on the plates are given only by the boundary part of the vacuum 
pressures.

Our calculations may be of interest in the brane-world cosmological scenarios. The braneworld corresponds to a manifold with dynamical boundaries and all fields which propagate in the bulk will give Casimir-type contributions to the vacuum energy, and as a result to the vacuum forces acting on the branes. In dependence of the type of a field and boundary conditions imposed, these forces can either stabilize or destabilize the brane-world. In addition, the Casimir energy gives a contribution to both the brane and bulk cosmological constant and, hence, has to be taken into account in the self-consistent formulation of the brane-world dynamics.( see for example [22, 36, 37, 38]).

\section{Acknowledgement}

I would like to thank Prof. A.A. Saharian for his comments and remarks.

\section{References}

[1] G. Plunien, B. Mueller, W. Greiner, Phys. Rep. 134, 87(1986).

[2] H. B. G. Casimir, proc. K. Ned. Akad. Wet. 51, 793(1948).

[3] E. Elizalde, S. D. Odintsov, A. Romeo, A. A. Bytsenko and S. Zerbini, zeta regularization techniques with applications(World Scientific, Singapore, 1994).

[4] E. Elizalde, Ten physical applications of spectral zeta functions, lecture notes in physics, (Springer-Verlage, Berlin, 1995).

[5] V. M. Mostepanenko and N. N. Trunov. The Casimir effect and its applications. (Oxford Science Publications New York, 1997).

[6] K. Kirsten, Spectral Functions in Mathematics and Physics, Chapman and Hall/CRC, Boca Raton, FL, 2001.

[7] M. Bordag, U. Mohideen, V.M. Mostepanenko, Phys. Rept. 353, 1, 2001.

[8] K. A. Milton, (2001). The Casimir Effect: Physical Manifestations of Zero-Point Energy (World Scientific, Singapore).

[9] M. Bordag, E. Elizalde and K. Kirsten, J. Math. Phys. 37, 895 (1996); M. Bordag, E. Elizalde, K. Kirsten and S. Leseduarte, Phys. Rev. D56, 4896 (1997); E. Elizalde, M. Bordag and K. Kirsten, J. Phys. A31, 1743 (1998).

[10] M. R. Setare and A. A. Saharian. Int. J. Mod. Phys. A16, 1463(2001).

[11] M. R. Setare and A. H. Rezaeian. Mod. Phys. Lett. A15, 2159(2000).

[12] M. R. Setare and R. Mansouri. Class. Quant. Grav. 18 (2001) 2331.

[13] M. R. Setare .Class. Quant. Grav. 18 (2001) 4823-4830. 
[14] S. A. Fulling, Phys. Rev D7, 2850 (1973).

[15] S. A. Fulling, J. Phys. A: Math. Gen. 10, 917 (1977).

[16] W. G. Unruh, Phys. Rev. D14, 870 (1976).

[17] D. G. Boulware, Phys. Rev. D11, 1404 (1975).

[18] R. M. Avagyan, A. A. Saharian, A. H. Yeranyan, Phys. Rev. D66, 085023, (2002).

[19] N. D. Birrell and P. C. W. Davies, Quantum fields in curved space,( Chambridge University press, 1986).

[20] P. Candelas and D. Deutsch, Proc. Roy. Soc. Lond. A 354, 79 (1977).

[21] M. R. Setare and R. Mansouri. Class. Quant. Grav. 18, 2659, (2001).

[22] A. A. Saharian, M. R. Setare, Phys. Lett. B552, 119, (2003).

[23] A. A. Saharian, Class. Quant. Grav. 19 5039, (2002) .

[24] P. Candelas and D. J. Raine, J. Math. Phys. 17, 2101 (1976).

[25] P. C. Davies and S. Fulling, Proc. Roy. Soc. Lond. A345, 59 (1977).

[26] P. Candelas and D. Deutsch, Proc. Roy. Soc. Lond. A362, 78 (1978).

[27] W. Troost and H. van Dam, Nucl. Phys. B159, 442 (1979).

[28] M. R. Brown and A. C. Ottewill, Phys. Rev. D31, 2514 (1985).

[29] M. R. Brown, A. C. Ottewill, and D. Page, Phys. Rev. D33, 2840 (1986).

[30] C. T. Hill, Nucl. Phys. B 277, 547 (1986).

[31] V. P. Frolov and E. M. Serebriany, Phys. Rev. D35, 3779 (1987).

[32] J. S. Dowker, Phys. Rev. D36, 3742 (1987).

[33] R. Parentani, Class. Quantum Grav. 10, 1409 (1993).

[34] V. Moretti and L. Vanzo, Phys. Lett. B375, 54 (1996).

[35] J. S. Dowker, R. Critchley, Phys. Rev. D13, 3224 (1976).

[36] M. R. Setare, Int. J. Mod. Phys. A18, 4285, (2003).

[37] M. R. Setare, hep-th/0308109, to be appear in Eur.Phys.J.C

[38] E. Elizalde, S. Nojiri, S. D. Odintsov, S. Ogushi, Phys. Rev. D67, 063515, (2003). 